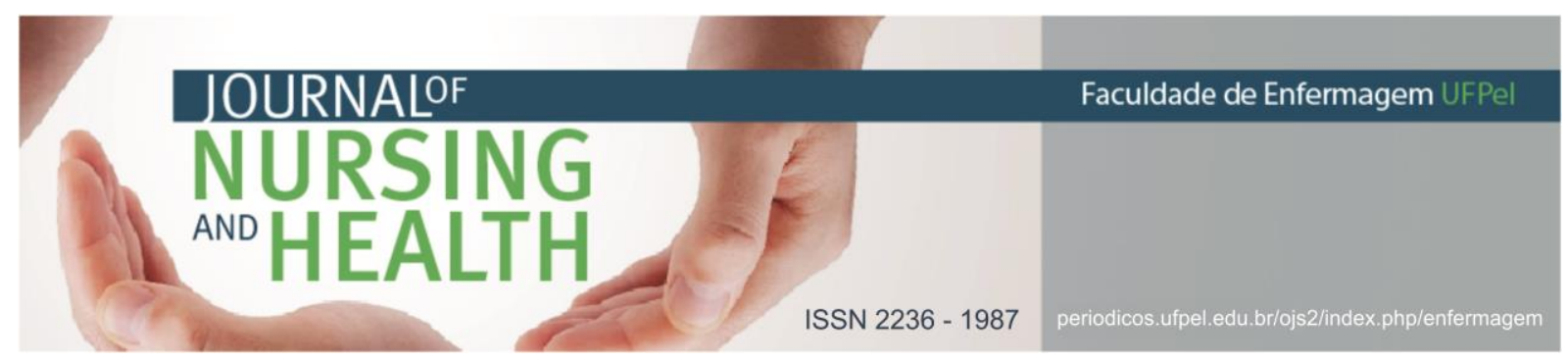

ARTIGO ORIGINAL

\title{
Percepção das nutrizes acerca do valor útil do apoio ao aleitamento materno
}

\author{
Nurturer's perception about the useful value of support for breastfeeding \\ Percepción de las nutrices acerca del valor útil del apoyo al alcance materno
}

Alves, Valdecyr Herdy ${ }^{1}$; Padoin, Stela Maris de Mello²; Rodrigues, Diego Pereira 3 ; Branco, Maria Bertilla Lutterbach Riker ${ }^{4}$; Marchiori, Giovanna Rosário Soanno5; Santos, Márcia Vieira dos ${ }^{6}$

Alves VH, Padoin SMM, Rodrigues DP, Branco MBLR, Marchiori GRS, Santos MV. Percepção das nutrizes acerca do valor útil do apoio ao aleitamento materno. J nurs health. 2018;8(3):e188306

\section{RESUMO}

Objetivo: analisar a valoração axiológica da mulher-nutriz quanto ao apoio no processo de lactação. Método: estudo de cunho social, exploratório, descritivo, qualitativo, realizado em maio e junho de 2014 no alojamento conjunto de um hospital universitário localizado em um município do Rio de Janeiro. Foram entrevistadas quinze mulheres-nutrizes internadas. Os dados coletados foram submetidos à análise de conteúdo na modalidade temática e à Teoria dos Valores, de Max Scheler. Resultados: o valor identificado foi o valor útil do apoio ao aleitamento materno e do seu manejo como processo para o enfrentamento das dificuldades vivenciadas pelas nutrizes no início da amamentação. Conclusão: a valoração da amamentação é importante estratégia para a atuação profissional, permitindo intervir direta e eficazmente junto às nutrizes com ações para assegurar o sucesso da promoção e do apoio ao aleitamento materno.

Descritores: Aleitamento materno; Valores sociais; Enfermagem; Enfermagem obstétrica.

\begin{abstract}
Objective: to analyze the axiological value of nurturing woman regarding the Objective: to analyze the axiological value of female nurturers regarding the support in the breastfeeding process. Methods: this is a Social, exploratory, descriptive, qualitative study, conducted between May and June 2014 in the joint accommodation of a university hospital in Rio de Janeiro. At the time, fifteen hospitalized nursing mothers were interviewed. The data were subjected to content analysis in thematic modality and Max Scheler's Theory of Values. Results: the useful value of breastfeeding support was identified and its management as a process for the confrontation with the difficulties experienced by nursing mothers at the early stages of breastfeeding were identified. Conclusion: the valuation of breastfeeding is an important strategy for health professional performance,
\end{abstract}

\footnotetext{
1 Enfermeiro. Doutor em Enfermagem. Universidade Federal Fluminense (UFF). E-mail: herdyalves@yahoo.com.br http://orcid.org/0000-0001-8671-5063

2 Enfermeira. Doutora em Enfermagem. Universidade Federal de Santa Maria (UFSM). E-mail: stelamaris_padoin@hotmail.com http://orcid.org/0000-0003-3272-054X

3 Enfermeiro. Mestre em Enfermagem. Centro Universitário Anhanguera de Niterói. E-mail: diego.pereira.rodrigues@gmail.com http://orcid.org/0000-0001-8383-7663

4 Enfermeira, Mestre em Materno-Infantil. Hospital Universitário Antônio Pedro da Universidade Federal Fluminense (UFF). E-mail: bertillariker@yahoo.com.br http://orcid.org/0000-0001-6307-4830

5 Enfermeira. Mestre em Saúde materno-infantil. Faculdade Novo Milênio. E-mail: giovannasoanno@gmail.com http: / / orcid.org/0000-0002-0675-1679

6 Enfermeira. Mestre em Saúde Materno-Infantil. Universidade Federal Fluminense (UFF). E-mail: enfa.marcia@oi.com.br http://orcid.org/0000-0002-1488-7314
} 


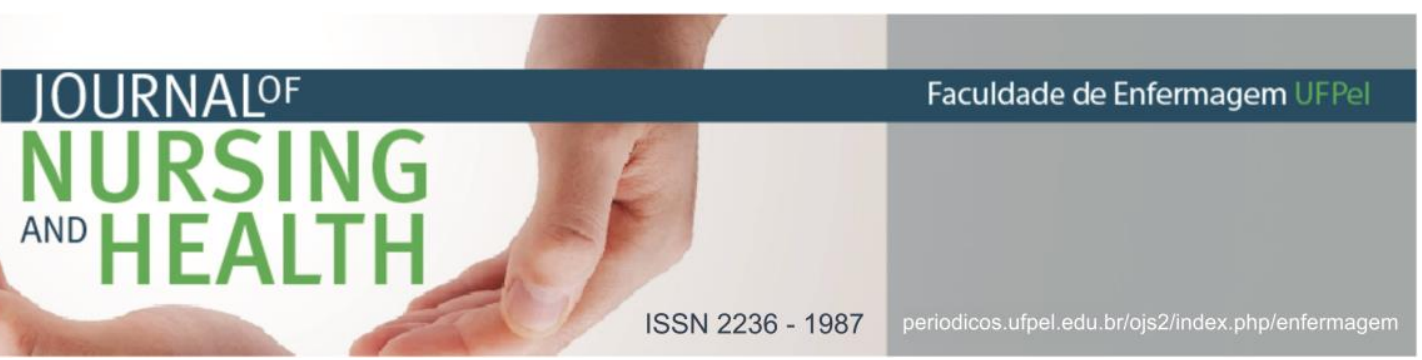

allowing direct and effective interventional on the mothers with actions to ensure the success of promotion and support to breastfeeding.

Descriptors: Breastfeeding; Social values; Nursing; Obstetric nursing.

\section{RESUMEN}

Objetivo: analizar el valor axiológico de la mujer educadora con respecto al apoyo en el proceso de lactancia materna. Método: este es un estudio social, exploratorio, descriptivo, cualitativo, realizado entre mayo y junio de 2014 en un hospital conjunto de un hospital universitario en Río de Janeiro. Quince mujeres madres lactantes fueron entrevistadas. Los datos se sometieron al análisis de contenido en modalidad temática y a la teoría de valores de Max Scheler. Resultados: el valor identificado fue el valor útil del apoyo a la lactancia materna y su manejo como un proceso de confrontación con las dificultades experimentadas por la madre lactante se identificó el inicio de la lactancia materna. Conclusión: el valor de la lactancia materna es una estrategia importante para el desempeño profesional, permite la intervención directa y efectiva entre las madres con acciones para garantizar el éxito de promoción y apoyo de la lactancia materna.

Descriptores: Lactancia materna; Valores sociales; Enfermería; Enfermería obstétrica.

\section{INTRODUÇÃO}

A prática do Aleitamento Materno (AM) tem sido defendida e apoiada no mundo todo como a melhor forma de nutrição exclusiva para o bebê até o sexto mês de vida e complementar até o segundo ano de vida. Além de trazer benefícios nutricionais por conter todos os nutrientes em qualidade e quantidade necessárias para o desenvolvimento adequado do lactente, o AM favorece e intensifica o vínculo entre mãe e filho. ${ }^{1}$

Apesar dos pontos positivos destacados e do conhecimento técnico-científico acumulado pelos profissionais de saúde, o desmame antes do período preconizado ainda é uma realidade que prevalece no Brasil. ${ }^{1}$ Nesse sentido, as estratégias de promoção, proteção e apoio ao AM tornam-se necessárias para romper com o processo do desmame precoce e auxiliar na saúde infantil. ${ }^{2}$

Nesse processo, é essencial que a equipe de saúde acolha mães e bebês e esteja disponível para a escuta e esclarecimento de dúvidas, incentive a troca de experiências com o intuito de atingir as recomendações do Fundo das
Nações Unidas para a Infância e da Organização Mundial da Saúde, que propõem o aleitamento exclusivo por seis meses e o complementado pelos alimentos da família, até os dois anos de idade ou mais. ${ }^{3-6}$

Estudo recente que analisou evidências científicas a partir de uma meta-análise, concluiu que as intervenções podem contribuir muito para promoção do AM. Essas consistiram em ações educativas e discussões face-a-face ou por meio expositivo ou ilustrativo, como o uso de folhetos e mídia eletrônica. Eram desenvolvidas no período gravídico puerperal distinto e pontual ou começando no período pré-natal e estendendo-se ao neonatal. ${ }^{7}$

Independente do período, pensar em uma intervenção de promoção do AM como prática assistencial sob o olhar das mulheres em processo de lactação, perpassa por uma questão de valores articulada no campo da vida, que envolve mulher/recém-nascido lactante, sua família e o profissional de saúde. Por isso, o prisma escolhido para compreender esse processo é o da 
ISSN 2236 - 1987

Axiologia, que significa valorar o $A M$ como questão humana e existencial, que não pode ser apreendida somente pelas experiências racionais, devendo estar atrelada às experiências do sentir valorativo de cada mulher no ato de ser nutriz.

Assim, para contemplar os múltiplos aspectos que envolvem as finalidades precípuas do apoio à amamentação, expressas na prática assistencial em saúde, teve-se como objetivo: analisar a valorização axiológica da mulher-nutriz quanto ao apoio no processo de lactação.

Foi adotado como base, o referencial teórico de Max Scheler. Para esse filósofo, a essência dos valores manifesta-se na experiência vivida dos seres humanos e assim, todas as coisas que nos cercam, por suas características próprias, possuem um determinado valor, um caráter próprio, particular, que faz com que se assuma diante delas uma escolha, mas nunca indiferença. ${ }^{8-11}$

\section{MÉTODO}

Estudo de cunho social, exploratório, descritivo, com abordagem qualitativa. Foi realizado no alojamento conjunto de uma maternidade universitária localizada na cidade de Niterói, Rio de Janeiro, com seus atendimentos destinados às usuárias do Sistema Único de Saúde (SUS) e à formação de profissionais de saúde no campo da saúde reprodutiva.

As puérperas internadas entre $1^{\circ}$ de maio e 30 junho de 2014 foram convidadas a participar do estudo e 15 foram entrevistadas, após terem assinado o Termo de Consentimento Livre e Esclarecido (TCLE) assegurando-lhes 0 anonimato mediante utilização de código alfanumérico representado pela letra $\mathrm{N}$ (Nutriz) seguido por um algarismo arábico (N1 a N15), conforme a sequência de realização das entrevistas, realizadas com roteiro semiestruturado. Foram critérios de inclusão na pesquisa: ser puérpera de risco habitual e estar em processo de lactação.

O corpus da pesquisa submetido à análise de conteúdo na modalidade temática, foi constituído pelas falas das participantes após terem sido gravadas com autorização das mesmas, transcritos e submetidos à regra de pertinência, permitindo a elaboração de dois eixos temáticos: ${ }^{12} 0$ reconhecimento da ajuda do profissional; as implicações positivas do manejo ao aleitamento materno.

Em conformidade com a Resolução $n^{\circ} 466$, de 12 de dezembro de 2012, do Conselho Nacional de Saúde, o estudo foi aprovado em 14 de abril de 2014 pelo Comitê de Ética em Pesquisa da Faculdade de Medicina da Universidade Federal Fluminense, sob Protocolo $n^{\circ}$ 615.070/2014.

\section{RESULTADOS}

\section{O reconhecimento da ajuda do profissional}

As nutrizes reconhecem a ajuda recebida dos profissionais no processo de amamentação. As dificuldades são superadas pelo desejo de amamentar e pela ajuda dos profissionais para acalmar a criança, colocá-la no peito e posicionar sua cabeça para a pega adequada: 
A posição para ele pegar no peito, ele não estava achando $e$ eu também não [...] assim, ele ficava muito irritado $e$ nem mamava direito, aí eu tive apoio aqui, as meninas me ajudaram, eu e ele ficamos tranquilos. N1

Ela demorou a pegar o peito para mamar, depois ficou tranquila, os profissionais de saúde me ajudaram. N7

Os depoimentos também evidenciaram o reconhecimento dos aspectos emocionais, técnicos e biológicos da amamentação:

Eu tive dificuldade [...]. As enfermeiras me ajudam, ficam perto. Agora acredito que ele vá mamar bem mais. N2

Eu pensei que eles iriam dar o NAN [...] eu tinha pouco leite, mas as enfermeiras disseram que é só estimular que o leite vem e deu certo. N3

Com o apoio, eu e o neném nos sentimos bem tranquilos $e$ a amamentação desceu mais rápido. N14

As implicações positivas do manejo ao aleitamento materno

A assistência no manejo clínico da amamentação com relação aos seus benefícios, incluindo as orientações para o aleitamento materno, encontrou eco nas palavras das nutrizes:

Eu fiquei ansiosa como colocar para mamar, as meninas me ensinaram a colocar no peito, deu certo, mas fazer ele (o bebê) acordar para mamar, ele não acordava de jeito nenhum, aí elas (a médica e a enfermeira) me ensinaram e deu certo. N10

Eu estava nervosa, elas vinham aqui e me ajudavam. E diziam: faz assim, e mostravam o peito, ensinando como é para o bebê pegar. Bom ter orientação, mesmo tendo outros filhos, porque é diferente. N11

0 apoio recebido no manejo clínico da amamentação, foi percebido pelas entrevistadas como sendo muito positivo para o sucesso da prática de aleitar:

Acho que a pior parte é quando o peito racha, não tive dificuldade no primeiro filho, mas agora está rachando, e elas (técnica de enfermagem e enfermeira) estão me ensinando a colocar o meu bebê para mamar, está aliviando a dor. N2

Elas ficam ajudando toda hora [...] são bem atenciosas, estão sempre dispostas a ajudar, assim fica mais tranquilo. N9

\section{DISCUSSÃO}

Quando percebemos ou captamos um valor de modo puramente intuitivo, isto é, o valor de toda aquisição que se refere a uma carência ou vontade de obter o objeto desejado, trata-se de um ato da consciência que intui o valor em direção ao desejável. A vida deve produzir algo útil apenas na medida em que se puder gozar daquilo que é agradável; ou seja, quando ela mesma é colocada como um dos valores vitais 


\section{JOURNALOF \\ NURSING \\ AND HEALTH}

ISSN 2236 - 1987

mais elevados e pode dominá-los livremente com um prazer constituído no ser humano. ${ }^{4,11}$

Os múltiplos e discrepantes posicionamentos em relação à natureza do valor, representados por correntes que integram a Teoria dos Valores, ou Axiologia, além da escassez de estudos com enfoque na presença e influência dos valores no ideário do aleitamento materno, levaram à necessidade de buscar autores cujas obras tratassem da análise valorativa, objetivando auxiliar o pesquisador na interpretação do conteúdo dos depoimentos obtidos a respeito do valor da amamentação. ${ }^{4,11,13}$

Percebe-se, nas falas das nutrizes, a sustentação fundamental da análise do valor que expressa a ideologia dominante relacionada aos desejos de amamentar e de ultrapassar as dificuldades cotidianas. Logo, a análise do valor do apoio à amamentação vai implicar na necessidade de se manter a noção de atenção valorativa (enquanto necessidade prática) como requisito prévio, indispensável à realização dos processos de cuidado em saúde, representado por uma assistência ética conforme a realidade de cada nutriz e pela possibilidade de transmitir segurança prática em relação ao ato de aleitar. ${ }^{4,11}$

0 enfermeiro deve apoiar o aleitamento com orientações quanto às diversas e confortáveis posições de amamentar, explicando as formas de reflexos da criança e mostrando como isso pode ser usado para ajudar na sucção. Torna-se essencial intervir nos obstáculos para o sucesso da amamentação, percebendo-o não apenas como um valor nutricional ou afetivo, mas também como um valor útil para a saúde da criança e da mulher, corroborando as diretrizes das políticas públicas voltadas para o AM. ${ }^{11,14}$ Além disso, para se constituir um valor em si mesmo, esse processo só é possível porque o outro (o profissional de saúde) disponibiliza à mulher-nutriz o seu apoio e ajuda para que obtenha sucesso ao amamentar, o que representa, na verdade, a superação da rotinização do discurso oficial proclamado cotidianamente nas instituições de saúde, cujo enfoque restringe-se ao valor biológico do leite humano, passando esse profissional a valorar os aspectos afetivos e emocionais da questão que envolvem a mulher-nutriz e a criança, garantindo o apoio à amamentação como valor útil.

Evidências científicas produzidas a respeito da amamentação, confirmam que a maioria dos programas oficiais de incentivo ao aleitamento já desenvolve ações educativas para as nutrizes, enfocando os aspectos emocionais, sociais, técnicos e biológicos da amamentação. ${ }^{4,7}$ Assim, tudo que significativamente se pode chamar de útil, é tomado apenas como meio para que o agradável se dê, como o ato de amamentar com prazer e sem dificuldades. O agradável é o valor fundamental; o útil, o valor derivado. 0 valor de toda e qualquer civilização que se paute pela utilidade, busca contemplar a plenitude de estar no mundo e, nesse sentido, também a sociedade atual busca o desfrute de coisas agradáveis como caminho para uma vida melhor. ${ }^{11,13}$ Portanto, os valores do agradável postos pelo valor utilitário, são bens da sociedade que 


\section{JOURNAIOF \\ NURSING \\ "No HEALTH \\ ISSN 2236 - 1987}

devem ser repartidos com os homens, visto que levam ao valor vital. ${ }^{13}$

A literatura científica enfoca os benefícios do apoio ao manejo clínico da amamentação. ${ }^{14-17}$ Desse modo, confirma 0 valor utilitário dessa assistência direcionada à nutriz frente aos seus valores. ${ }^{11,13}$ Assim, a assistência inclui técnicas e orientações específicas ofertadas pelas equipes de saúde envolvidas com a saúde da criança e da mulher. ${ }^{16,18}$

Nota-se que os depoimentos desvelam o valor útil desse processo como um valer prático, que resulta em valor vital como possibilidade de superar a dicotomia já referida, em busca das dimensões subjetivas/objetivas contidas nos discursos das entrevistadas que revelam, não só o fenômeno valor útil mas, também, a sua relação com a amamentação quando realizada de forma agradável e prazerosa. ${ }^{11,13}$ Essa é a questão embutida nos discursos das nutrizes que, ao se envolverem realmente com 0 valor $\mathrm{da}$ amamentação, valoram o seu pensar e o seu sentir na plenitude desse ato, passando a vivenciar valores que refletem a ação levada a efeito por uma pessoa (o profissional de saúde) que, por sua vez, possibilita a realização de outra pessoa (a mulhernutriz), corroborando a literatura consultada no sentido de que todo valor sempre remete a outro valor que lhe dá uma realidade significativa. 4,15

Os primeiros dias após o parto são cruciais para o aleitamento materno ser bem-sucedido, pois é quando a lactação se estabelece, além de ser um período de intenso aprendizado para a mãe e de adaptação para o recém- nascido. Várias dúvidas e problemas podem surgir e tornar a mulher vulnerável e insegura, sendo relevante que os profissionais de saúde reconheçam e demonstrem valorar as demandas apresentadas por ela, favorecendo uma assistência de saúde útil, informando com clareza as estratégias e vantagens de iniciar e dar continuidade ao processo de aleitamento materno. ${ }^{18}$ Para tanto, é imprescindível investir em atividades como visitas domiciliares, palestras, grupos de apoio e aconselhamento para incentivo e manutenção do aleitamento exclusivo, a fim de intensificar as ações promovidas durante 0 período de pós-parto hospitalar. ${ }^{4,14-15,18}$

Nesse sentido, como o sucesso da amamentação não depende só da nutriz e de seu bebê, o profissional de saúde (no caso, o enfermeiro) passa a ser reconhecido como sujeito que se abre para a experiência do outro, incorporando as diferenças entre ambos e assumindo uma assistência ética e valorativa ao perceber que assisti-la é possível, sendo necessário haver um processo de valoração entre ambos, assim garantindo uma assistência útil de fato para que alcancem o valor do agradável: no caso da mulher-nutriz, por ser atendida em suas demandas de saúde; e do profissional, por tê-la assistido eticamente.

Assim, na Axiologia, quando se valora, considera-se não apenas o que está dito, mas também o que está implícito e, portanto, está significando. ${ }^{11}$ Logo, o valor útil do apoio à amamentação preenche uma carência vivenciada pelas nutrizes na 
prática de amamentar, cuja valoração quanto à assistência útil confirma-se como forma de valor para o manejo clínico da amamentação. ${ }^{4,13}$

Por fim, o valor útil se apresentou no que diz respeito ao apoio à amamentação no espaço da maternidade do hospital universitário em foco, apoio valorado quando é reconhecido pela nutriz como um preenchimento do desejo de amamentar sua cria; caso contrário, será uma desvalorização do valor da amamentação, ou seja, um não valor por não atender as suas demandas. Nesse sentido, para o sucesso da amamentação, deve-se garantir uma assistência útil de fato, que alcance o valor do agradável em um cuidado ético que permita à mulher ser atendida em todas as suas demandas carenciais.

\section{CONCLUSÃO}

Foi possivel perceber que as entrevistadas enfatizaram a importância do apoio recebido durante a prática profissional, especialmente na maternidade do hospital universitário. Isso se revelou como um valor vital para elas, desde que fossem compreendidas em seu respectivo existir concreto e histórico. Seus discursos apresentaram-se impregnados pelos valores utilitários da amamentação, decorrentes do processo de apoio que vivenciaram durante a internação na maternidade. A experiência também foi considerada um valor útil para o momento advindo da alta hospitalar, para a garantia do sucesso da amamentação no contexto de vida domiciliar e cotidiano.
Concluímos que a teoria dos valores se constitui um importante subsídio para a enfermagem, uma vez que permite uma abordagem estratégica na atuação profissional, admitindo intervir direta e eficazmente junto às nutrizes com ações para assegurar o sucesso da promoção e do apoio ao aleitamento materno.

\section{REFERÊNCIAS}

1 Leal CCG, Fonseca-Machado MO, Oliveira LCQ, Monteiro JCS, Leite AM, Gomes-Sponholz FA. Prática de enfermeiras na promoção do aleitamento materno de adolescentes brasileiras. Cienc enferm [Internet]. 2016 [acesso em 2018 set 24];22(3):97$106 . \quad$ Disponível em: https://scielo.conicyt.cl/pdf/cienf/v2 2n3/0717-9553-cienf-22-03-00097.pdf

2 Battaus MRB, LiberaliR. A promoção do aleitamento materno na estratégia de saúde da família - revisão sistemática. Rev APS [Internet]. 2014 [acesso em 2018 set 24];17(1):93-100. Disponível em: https://aps.ufjf.emnuvens.com.br/ap s/article/view/1843/789

3 Almeida JM, Luz SAB, Ued FV. Support of breastfeeding by health professionals: integrative review of the literature. Rev paul pediatr [Internet]. 2015 [cited 2018 set 24];33(3):355-62. Available from: http://www.scielo.br/pdf/rpp/v33n3 /en_0103-0582-rpp-33-03-0355.pdf

4 Alves $\mathrm{VH}$, Rodrigues DP, Gregório VRP, Branco MBLR, Souza RMP, Alves CMCSH. Reflexions about the value of breastfeeding as a health practice: a nursing contribution. Texto \& contexto enferm [Internet]. 2014 [cited 2018 


\section{JOURNALOF \\ NURSING \\ ANO HEALTH}

ISSN 2236 - 1987

Sept 24];23(1):203-10. Available from: http://www.scielo.br/pdf/tce/v23n1/ 0104-0707-tce-23-01-00203.pdf

5 Aparecida KRM, Chaves LC, Filipini R, Fernandes IC. Percepção das mães em relação ao aleitamento materno no período do pós-parto. ABCS health sciences [Internet]. 2014 [acesso em 2018 set 24];39(3):146-52. Disponível em:

https: //portalnepas.org.br/abcshs/art icle/view/648/647

6 Caniçali Primo C, Dutra PR, Lima EFA, Alvarenga SC, Leite FMC. Redes sociais que apoiam a mulher durante a amamentação. Cogitare enferm [Internet]. 2015 [acesso em 2018 set 24];20(2):426-33. Disponível em: http: / / revistas.ufpr.br/cogitare/articl e/view/37453/25554

7 Mahesh PKB, Gunathunga MW, Arnold SM, Jayasinghe $C$, Pathirana S, Makarim $M F$, et al. Effectiveness of targeting fathers for breastfeeding promotion: systematic review and meta-analysis. BMC public health [Internet] 2018 [cited 2018 Oct 04]; 24;18(1):1140. Available from: https: / /bmcpublichealth.biomedcentr al.com/articles/10.1186/s12889-0186037-x

8 Scheler M. Ética. Nuevo ensayo de fundamentación de un personalismo ético. Buenos Aires: Caparrós editores; 2001.

9 Scheler M. Ensayos de una filosofia de la vida. Buenos Aires: Nova; 1960.

10 Scheler M. Der formalismus in der ethik und die materielle wertethik. Adamant Media Corporation: Bouvier; 2004.
11 Scheler M. Da reviravolta dos valores. 2a ed. Petrópolis: Vozes; 2012.

12 Câmara RH. Análise de Conteúdo: da teoria à prática em pesquisas sociais aplicadas às organizações. Gerais (univ fed Juiz Fora) [Internet]. 2013 [acesso em 2018 set 24];6(2):179-91. Disponível em: http://pepsic.bvsalud.org/pdf/gerais/ v6n2/v6n2a03.pdf

13 Alves VH, Rodrigues DP, Cabrita BAC, Vieira BDG, Branco MBLR, Sá AMP. Breastfeeding as an evaluative practice in know-how: a descriptive study. Online braz j nurs [Internet]. 2013 [cited 2018 Sept 24]; 12(4):902-10. Available from: http: / / www.objnursing.uff.br/index. php/nursing/article/view/4154/pdf_3 6

14 Baptista SS, Alves VH, Souza RMP, Rodrigues DP, Barbosa MTSR, Vargas GSA. The lactation in women with premature babies: reconstructing the nursing care. Rev pesqui cuid fundam [Internet]. 2014 [cited 2018 Sept 24];6(3):1036-46. Available from: http://www.seer.unirio.br/index.php /cuidadofundamental/article/view/32 05/pdf_1352

15 Azevedo ARR, Alves VH, Souza RMP, Rodrigues DP, Branco MBLR, Cruz AFN. Clinical management of breastfeeding: knowledge of nurses. Esc anna nery rev enferm [Internet]. 2015 [cited 2018 Sept 24];19(3):439-45. Available from: http://www.scielo.br/pdf/ean/v19n3 /en_1414-8145-ean-19-03-0439.pdf

16 Souza RMP, Alves VH, Rodrigues DP, Branco MBLR, Lopes FO, Barbosa MTRS. Nursing strategies in the clinical management of breastfeeding: a descriptive and exploratory study. 


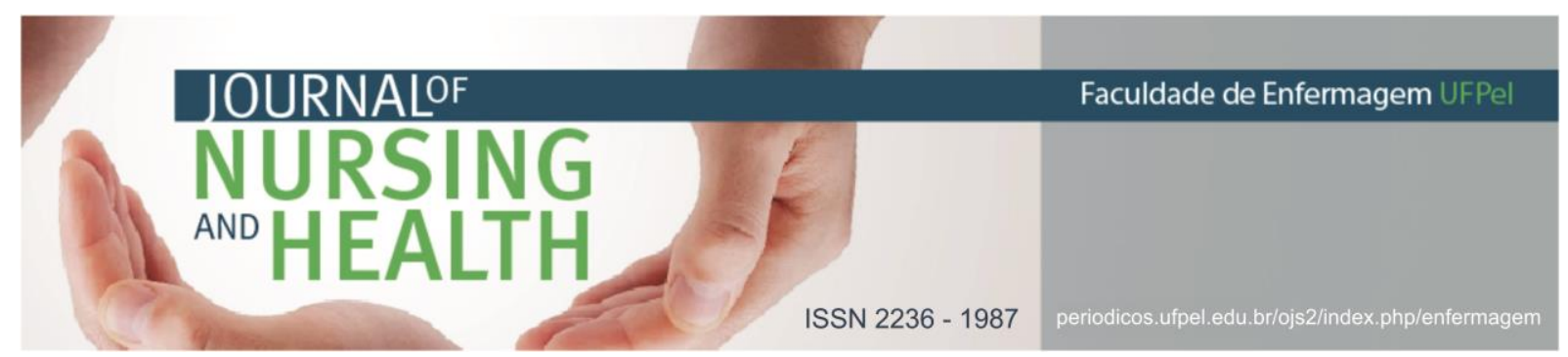

Online braz j nurs [Internet]. 2015 [cited 2018 Sept 24];14(1):51-61. Available from: http://www.objnursing.uff.br/index. php/nursing/article/view/4612/pdf_3 67

17 Santos LGB, Nascimento LCN, Leite FMC, Moser DC. Manejo do aleitamento materno: ações de enfermeiros das equipes de Estratégias de Saúde da Família de um município do norte do Espírito Santo. Rev bras pesqui saúde [Internet]. 2014 [acesso em 2018 set 24];16(1):8-15. Disponível em: http://periodicos.ufes.br/RBPS/articl e/view/8487/5983

18 Santos FCS, Cyrino ACT, Santos FS, Santos Neto M, Abrahão FNA. Atuação dos enfermeiros em unidades básicas de saúde amigas da amamentação. Rev rene (online) [Internet]. 2014 [acesso em 2018 set 24];15(1):70-7. Disponível em:

http://www.periodicos.ufc.br/rene/a rticle/view/3084/2369

Data de submissão: 17/08/2018

Data de aceite: 07/10/2018

Data de publicação: 12/11/2018 